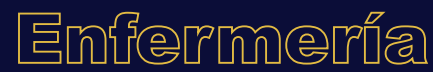

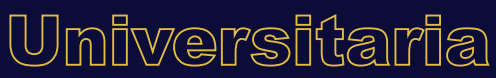

\section{La simulación computarizada, herramienta didáctica para el desarrollo de habilidades en la valoración del bienestar materno-fetal}

\author{
Computer simulation, a didactic tool \\ for the development of skills in the \\ assessment of maternal-fetal well-being
}

\section{Simulação computadorizada: ferramenta didática para o desenvolvimento de habilidades na avaliação do bem-estar materno fetal}

\author{
M.N. Posada-Morales ${ }^{a{ }^{*}}$, M.N. Muñoz-Astudillo ${ }^{b 1}$, \\ P. Durán-Ospina ${ }^{c_{2}}$, D.F. Ramírez-Moncayo ${ }^{d_{3}}$ \\ ORCID \\ ${ }^{\mathrm{a}} 0000-0002-4326-1177$ \\ c0000-0002-5758-3775 \\ ${ }^{\mathrm{b}}$ 0000-0002-9310-3310 \\ d0000-0003-1776-8764 \\ ${ }^{1}$ Fundación Universitaria del Área Andina, Programa de Enfermería, Risaralda, Pereira, Colombia \\ ${ }^{2}$ Universidad Técnica de Manabí, Portoviejo, Manabí, Ecuador \\ ${ }^{3}$ Tecnoparque Sena, Nodo Pereira, Colombia
}

Recibido: 20 mayo 2020

Aceptado: 25 febrero 2021

RESUMEN

Introducción: Las tecnologías de información y comunicación están incorporadas a la práctica docente en enfermería materno-perinatal e implican un reto permanente en generación de herramientas didácticas para facilitar el desarrollo de habilidades en la valoración del bienestar materno-fetal. Con fundamento en la teoría del aprendizaje experiencial de David Kolb y en la filosofía de principiante a experto de Patricia Benner se planteó como

*Autora para correspondencia. Correo electrónico: mposada@areandina.edu.co https://doi.org/10.22201/eneo.23958421e.2021.1.894

1665-7063/@ 2021 Universidad Nacional Autónoma de México, Escuela Nacional de Enfermería y Obstetricia. Este es un artículo Open Access bajo la licencia CC BY-NC-ND (http://creativecommons.org/licenses/by-nc-nd/4.o/). 
objetivo diseñar e implementar un simulador computarizado de fidelidad media para reforzar el aprendizaje en la interpretación del trazado toco-cardiográfico.

Desarrollo: Proyecto de desarrollo tecnológico en tres fases: 1. Conformación del equipo de diseño necesario para trabajar requerimientos funcionales y presentación del simulador, 2. Fundamentación pedagógico-didáctica disciplinar y metodológica para implementar requerimientos científico-técnicos, 3. Creación del simulador computacional con funciones de aprendizaje, evaluación para estudiantes y docentes; tutoriales, e interfaz al servidor institucional.

Se creó e implementó un simulador computacional con grado de maduración TRL6 que logró reproducir el círculo de aprendizaje consistente en: Experiencia concreta, Observación reflexiva, Conceptualización abstracta y Experimentación activa; componentes relacionados con un proceso complejo de retroalimentación permanente y repetición, hasta aprender a valorar el bienestar materno-fetal a partir de la interpretación del trazado toco-cardiográfico asociado a la situación clínica. La evaluación cualitativa de la experiencia mostró adecuada usabilidad del simulador.

Conclusiones: La simulación computarizada constituye una valiosa herramienta didáctica, un entorno de aprendizaje que logra integrar dimensiones pedagógicas, conceptuales, disciplinares y tecnológicas, pero el cual requiere la presencia del docente quien direcciona la experiencia para garantizar el aprendizaje.

Palabras clave: Simulación por computador; tecnología educacional; enfermería; competencia clínica; atención perinatal; monitoreo fetal; Colombia.

\section{ABSTRACT}

Introduction: Information and Communication Technologies are incorporated into the teaching practice in maternal-perinatal nursing and imply a permanent challenge in the generation of didactic tools to facilitate the development of skills in the assessment of maternal-fetal well-being. Based on the theory of Experiential Learning by David Kolb and the philosophy of Beginner to Expert by Patricia Benner, the objective was to design and implement a medium fidelity computerized simulator to reinforce learning in the interpretation of the tocho-cardiographic tracing.

Developing: Technological development project, in three phases: 1. Creation of the design team necessary to work on functional requirements and presentation of the simulator. 2 . Pedagogical-didactic disciplinary and methodological foundation: to implement scientific-technical requirements. 3. Creation of the computational simulator with learning and evaluation functions for students and teachers; tutorials, and interface to the institutional server.

A computational simulator with a TRL6 degree of maturity was created and implemented, which managed to reproduce the learning circle consisting of: Concrete experience, Reflective observation, Abstract conceptualization and Active experimentation. Related components in a complex process of permanent and repetitive feedback, until learning to assess the maternal-fetal well-being from the interpretation of the tocho-cardiographic tracing associated with the clinical situation. The qualitative evaluation of the experience showed adequate usability of the simulator. 
Conclusions: Computer simulation constitutes a valuable didactic tool, a learning environment that manages to integrate pedagogical, conceptual, disciplinary and technological dimensions, but requires the presence of the teacher who directs the experience to guarantee learning.

Keywords: Computer simulation; educational technology; nursing; clinical competence; perinatal care; fetal monitoring; Colombia.

\section{RESUMO}

Introdução: As Tecnologias de Informação e Comunicação estão incorporadas à prática docente em enfermagem materno-perinatal e implicam em desafio permanente na geração de ferramentas didáticas que facilitem o desenvolvimento de habilidades na avaliação do bem-estar materno-fetal. Com base na teoria de Aprendizagem Experiencial de David Kolb e na filosofia de Beginner to Expert de Patrícia Benner, o objetivo foi projetar e implementar um simulador computadorizado de média fidelidade para reforçar o aprendizado na interpretação do traçado toco-cardiográfico.

Desenvolvimento: Projeto de desenvolvimento tecnológico, em três fases: 1. Criação da equipe de design necessária para trabalhar os requisitos funcionais e apresentação do simulador. 2. Fundamentação disciplinar e metodológica pedagógico-didática: implementar requisitos técnico-científicos. 3. Criação do simulador computacional com funções de aprendizagem e avaliação para alunos e professores; tutoriais e interface com o servidor institucional.

Foi criado e implementado um simulador computacional com grau de maturidade TRL6, que conseguiu reproduzir o círculo de aprendizagem constituído por: Experiência concreta, Observação reflexiva, Conceituação abstrata e Experimentação ativa. Componentes relacionados em um complexo processo de feedback permanente e repetitivo, até aprender a avaliar o bem-estar materno-fetal a partir da interpretação do traçado toco-cardiográfico associado à situação clínica. A avaliação qualitativa da experiência mostrou adequada usabilidade do simulador.

Conclusões: A simulação computacional constitui uma valiosa ferramenta didática, um ambiente de aprendizagem que consegue integrar as dimensões pedagógica, conceitual, disciplinar e tecnológica, mas requer a presença do professor que direciona a experiência para garantir a aprendizagem.

Palavras chave: Simulação por computador; tecnologia educacional; enfermagem; competência clínica; assistência perinatal; monitorização fetal; Colômbia.

\section{INTRODUCCIÓN}

\section{Los simuladores}

Para fortalecer las competencias profesionales es fundamental innovar en métodos y técnicas de aprendizaje; de ahí que el uso de las tecnologías de la información y comunicación (TIC) en lo cotidiano de la Educación Superior sea imprescindible ${ }^{1,2}$. La práctica formativa con simuladores se utiliza en todas las ciencias de la salud por considerarse de bajo riesgo y protectora de la seguridad del paciente 3.4 .

Cuando se utiliza el término simulador, generalmente se hace referencia a equipos que se utilizan para incrementar la destreza individual en condiciones controladas. La simulación clínica 
incluye diversos tipos de equipos: maniquís, equipos robóticos, realidad virtual, sistemas inteligentes y simuladores computacionales, entre otros ${ }^{5-7}$. De acuerdo con $\mathrm{Casal}^{8}$, unos u otros simuladores se utilizan según las competencias que se pretendan desarrollar o fortalecer.

El uso de entornos computarizados tiene dos grandes ventajas: la accesibilidad, que es la posibilidad de acceder desde cualquier lugar a través de dispositivos móviles, y la disponibilidad que ofrecen los proveedores de servicios en la nube, pues el tiempo en línea generalmente oscila entre 99.5\% y 99.9\%. Estas propiedades son diferenciadoras en el contexto de aprendizaje y han impulsado la generación de simuladores clínicos computacionales.

La introducción de la simulación computarizada tiene una influencia favorable en el proceso de aprendizaje ${ }^{9}$. Los estudiantes tienen actitudes positivas y proactivas hacia este tipo de TIC $^{10}$ y por su componente multisensorial abre espacios para la interacción con datos, gráficos, imágenes, audios y registros, que, desde el punto de vista de la neurolingüística, incrementan las posibilidades para asimilar y comprender la nueva información ${ }^{11}$. Estas características unidas a una secuencia lógica, relevante y dinámica del contenido llevan a la activación de conocimientos previos, desarrollo de habilidades, aplicación de procesos y operaciones mentales (análisis, inferencias, juicio crítico y otras), requeridos para el aprendizaje?.

\section{La simulación computarizada en la formación de Enfermería}

En los programas educativos de enfermería se han incorporado simuladores computacionales que abren la posibilidad no sólo de interactuar con la información, sino de realizar prácticas sistemáticas para reforzar conocimientos, técnicas y procedimientos de modo que se logren habilidades en todas las áreas de formación ${ }^{5,12-14}$. En estos escenarios el aprendizaje se convierte en un proceso complejo que demanda repetir experiencias, observaciones y conceptualizaciones para orientar la acción.

Sin lugar a duda, el desarrollo de habilidades procedimentales [prácticas] en un estudiante de enfermería es de gran relevancia para el ejercicio profesional, personal y académico. Por lo tanto, la simulación computarizada y con escenarios de alta fidelidad han cobrado sentido al demostrar que lo aprendido por estos recursos es más motivador y se transfiere con mayor eficacia a una situación real que lo aprendido por otros medios ${ }^{15}$; lo que posiblemente se deba al componente multisensorial y psicológico que involucran.

De hecho, al diseñar y crear un simulador computacional debe existir la intencionalidad de acercarse a un determinado fenómeno de estudio y reproducirlo en sus características esenciales para que el estudiante comprenda mejor la situación que se le presenta además de que establezca inferencias y asociaciones necesarias para el aprendizaje ${ }^{16}$.

Acorde con lo anterior, vale la pena mencionar algunos simuladores computacionales desarrollados en enfermería. Por ejemplo, Araújo Girão et al. ${ }^{17}$, crearon un software de juego virtual con la intención de que los estudiantes de enfermería pudieran preparar y administrar medicamentos. Para ello integraron personajes, ambientes y actividades con los cuales debía interactuar el alumno asumiendo su papel de cuidador. Una vez validado se demostró que fue ideal para comprender la complejidad del proceso y útil para crear transferibilidad. Aunado a esto, se ha corroborado el potencial de los simuladores computacionales en el proceso de enseñanza-aprendizaje y en la aplicación de conocimientos ${ }^{18}$.

Por su parte, Avari et al. ${ }^{19}$ crearon un software para entrenamiento en cálculo de dosis de medicamentos; el diseño involucró aspectos multisensoriales: gráficos, galería de imágenes, texto, audio y un álbum de películas, cuyas particularidades impactan en la estructura cerebral y la distribución 
sináptica, mecanismos donde yacen los procesos de aprendizaje ${ }^{20} \mathrm{y}$ demuestran la importancia de los motivadores en la construcción de los softwares educativos.

Sin embargo, esta clase de productos tecnológicos tienen altos costos e inversión de tiempo, motivo por el cual muchos simuladores computacionales no avanzan de su estado de prototipos, es decir, simuladores funcionales con requerimientos básicos ${ }^{21}$. Por consiguiente, es fundamental la creación de un simulador computacional para poner en práctica los conocimientos sobre valoración del bienestar materno-fetal a través de la interpretación del trazo de monitorización fetal, siguiendo una ruta de aprendizaje a partir de la experiencia.

Las características y las tareas propuestas en el simulador deben estar organizadas dentro de una estructura secuencial, básicamente porque los estudiantes novatos comprenden mejor un fenómeno que se les presenta en forma ordenada, coherente y en función de su disciplina. El alumno debe iniciar la interacción con el simulador teniendo conocimiento de la meta que persigue, para ello la puerta de entrada es la valoración clínica del sujeto de cuidado enfermero; el análisis de la situación de salud le permitirá comprender la magnitud del problema y conducir a su resolución, con base en algunos criterios clínicos.

Al considerar que las habilidades y el juicio crítico se desarrollan progresivamente hasta consolidarse en la transferibilidad ${ }^{22}$, es necesario disponer de una herramienta válida, confiable, factible, de fácil acceso, que sirva de apoyo, lo motive, le permita repetir el procedimiento tantas veces como lo requiera y le garantice al estudiante un aprendizaje efectivo. La creación de un simulador computacional tiene como premisa una práctica fuertemente vinculada con el aprendizaje de los conocimientos y por tal motivo se reconoce que entre más se practique, más posibilidad se tiene de aprender, sobre todo de los errores, lo cual nos lleva a inferir que entre más experiencias simuladas se tengan, mayor habilidad se adquiere ${ }^{6}$.

\section{Fundamentación pedagógico-didáctica y disciplinar}

Las herramientas tecnológicas se convierten en didácticas y adquieren sentido pedagógico cuando tienen como propósito la formación del ser, saber y hacer, en un contexto específico de aprendizaje, a través de procesos de innovación educativa ${ }^{23}$, los cuales están permeados por fundamentos pedagógicos-didácticos, conceptuales, disciplinares y tecnológicos, orientados hacia un aprendizaje que adquiere significado en la práctica, sea esta en escenarios simulados o reales.

Desde una perspectiva pedagógico-didáctica, el aprendizaje experiencial ${ }^{24}$ ofrece fundamentos para la simulación computarizada. Para un aprendizaje efectivo, David Kolb, citado por Gómez²4, propone un proceso en cuatro etapas: 1. Se tiene una experiencia concreta; 2. Observación reflexiva. Se reflexiona sobre la experiencia, estableciendo conexión entre lo que se hace y lo que se obtiene; 3 . Conceptualización abstracta. Se sacan conclusiones o generalizaciones sobre lo reflexionado; y 4 . Experimentación activa. Se pone en práctica las conclusiones obtenidas. La secuencia planteada por Kolb, según Gómez ${ }^{24}$, no siempre sigue el mismo orden, pues muchas veces se utilizan reflexiones derivadas de experiencias anteriores y conocimientos previos.

El abordaje del aprendizaje experiencial para la enseñanza del cuidado enfermero tiene sustento en la filosofía de Benner, citada por Carrillo Algarra ${ }^{25}$. Esta filosofía reconoce que en el proceso educativo se deben integrar diferentes prácticas experimentales en el enfermero aprendiz para que progresivamente adquiera mayor experticia, lógicamente, enmarcadas en los avances científicos, técnicos, tecnológicos y conceptuales. 
Uno de los retos en la enseñanza de los fundamentos conceptuales de la enfermería es la valoración del bienestar materno-fetal; en la actualidad se dispone de la monitorización electrónica, la cual debe ser interpretada correctamente por los enfermeros para tomar decisiones oportunas ${ }^{26}$. En ese sentido, se requiere reforzar la práctica de los estudiantes para el desarrollo de habilidades en la valoración clínica e inferir la existencia o no de bienestar, hecho que se dificulta puesto que la prueba se basa en la identificación visual de ciertos patrones del trazado ${ }^{27}$. Por consiguiente, es necesario que el estudiante interactúe con muchas situaciones de salud ligadas a un registro de monitorización materno-fetal para lograr desarrollar dichas habilidades.

Conscientes de que el alumno desde su condición de novato necesita tener varias experiencias simuladas que gradualmente lo lleven a la experticia, se propuso crear un simulador computacional, con requerimientos básicos, funcionales y elementos multimedia que crean un escenario acorde al real. Más aún, se espera que el estudiante interactúe con el sujeto de cuidado (gestante), utilice operaciones mentales para analizar la situación de salud simulada y asocie e interprete el trazado de monitorización fetal que se le presenta, de modo que pueda inferir la existencia de bienestar o no bienestar fetal, y establecer a su juicio crítico, las medidas que serían necesarias en el proceso de cuidado.

\section{DESARROLLO}

\section{Diseño y creación del simulador computarizado}

El desarrollo tecnológico se llevó a cabo en tres fases:

- Fase 1. Exploratoria: Tuvo una duración de un mes en la que se conformó un equipo de diseño con seis integrantes: dos docentes enfermeras especialistas del área materno perinatal con experiencia en didáctica, un gestor de proyectos en la línea de tecnologías virtuales educativas, un programador y dos estudiantes de enfermería. Teniendo en cuenta aspectos conceptuales, disciplinares, pedagógicos y tecnológicos, se plantearon ideas creativas que serían implementadas en el simulador computacional.

- Fase 2. Fundamentación conceptual y metodológica: Desarrollada durante tres meses. Se definió contenido, operaciones mentales, forma de evaluación y retroalimentación, elementos motivadores, entorno multisensorial, sistema de navegación, actividades interactivas, secciones para acceso y secuencia de las interfaces para dar cuerpo a los elementos de la teoría del aprendizaje experiencial y los elementos de la filosofía de Benner citada por Carrillo Algarra ${ }^{25}$ que sustentan la práctica enfermera.

- Fase 3. Creación del simulador computacional: Esta etapa duró ocho meses y consistió en la construcción del simulador atendiendo los requerimientos específicos del proceso de aprendizaje.

\section{Características tecnológicas del simulador}

Condiciones técnicas: El simulador está recreado con ilustraciones, diseños e interfaces (pantallas) que muestran ordenadamente las siguientes ventanas: inicio, ingreso de estudiantes, situación de salud expresada en la historia clínica, trazado de la actividad cardíaca fetal, categorías de interpretación del trazado, audios de retroalimentación y rúbrica del desempeño del estudiante. El acceso se realiza a través de la página web institucional, compatible con navegadores de PC y móviles. 
Características pedagógico-disciplinares: El simulador muestra una secuencia de aprendizaje y su relación con las actividades de valoración del bienestar materno-fetal, basadas en Kolb, según lo descrito por Gómez ${ }^{24}$ :

1. Percepción de información a través del sujeto de cuidado: La sección de inicio del simulador (Figura 1) contiene imágenes de la diada materno-fetal. La figura humana tiene un gran valor psicológico porque conforma un referente social y emocional importante en el aprendizaje y en la motivación para realizar la práctica que se propone. Se busca que estas imágenes reflejen sentimientos gratos, faciliten el pensamiento conceptual y la memorización ${ }^{28}$.

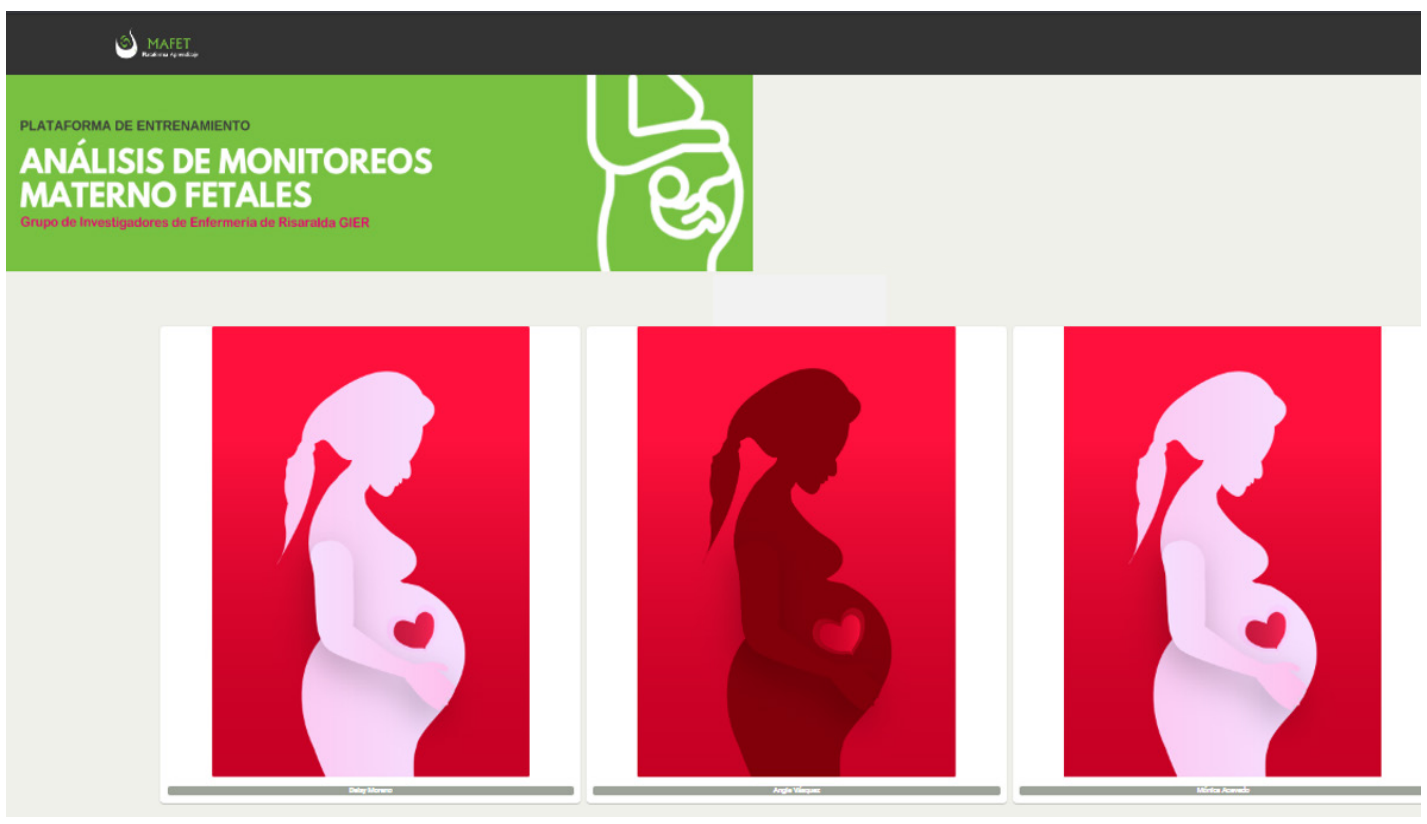

Figura 1. Sección: Inicio del simulador computacional

2. Procesamiento de la información: Se presenta la situación de salud de la gestante (Figura 2) y un trazado de monitorización materno-fetal (Figura 3), con el fin de ser analizados. Involucra operaciones mentales para organizar la información, interpretarla, hacer inferencias, asociaciones y, finalmente, emitir juicios críticos ${ }^{29}$, en relación con los hallazgos de la monitorización materno-fetal ${ }^{30}$

3. Aplicación de la información: En esta sección el simulador muestra las categorías de interpretación de la monitorización materno-fetal (Figura 4). El estudiante, de acuerdo con los patrones de frecuencia cardiaca fetal y el análisis de la situación de salud, determina el estado de bienestar fetal o las posibles alteraciones para deducir la necesidad de intervención. 
4. Reflexión sobre la experiencia: Para atender este componente el simulador dispone de una rúbrica que permite al estudiante hacer el seguimiento detallado de su desempeño (Figura 5). Este aspecto es importante en el desarrollo de competencias emocionales, ya que la retroalimentación y la evaluación formativa llevan a la autorreflexión y esta a su vez conduce a identificar los errores como una experiencia de aprendizaje, lo que facilita el cambio ${ }^{31}$.

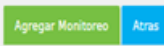

Ingrese el nombre de la paciente

Ingrese la historia clínica

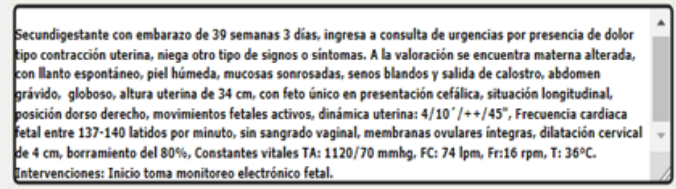

STCushite

Figura 2. Sección: Situación de salud de la gestante
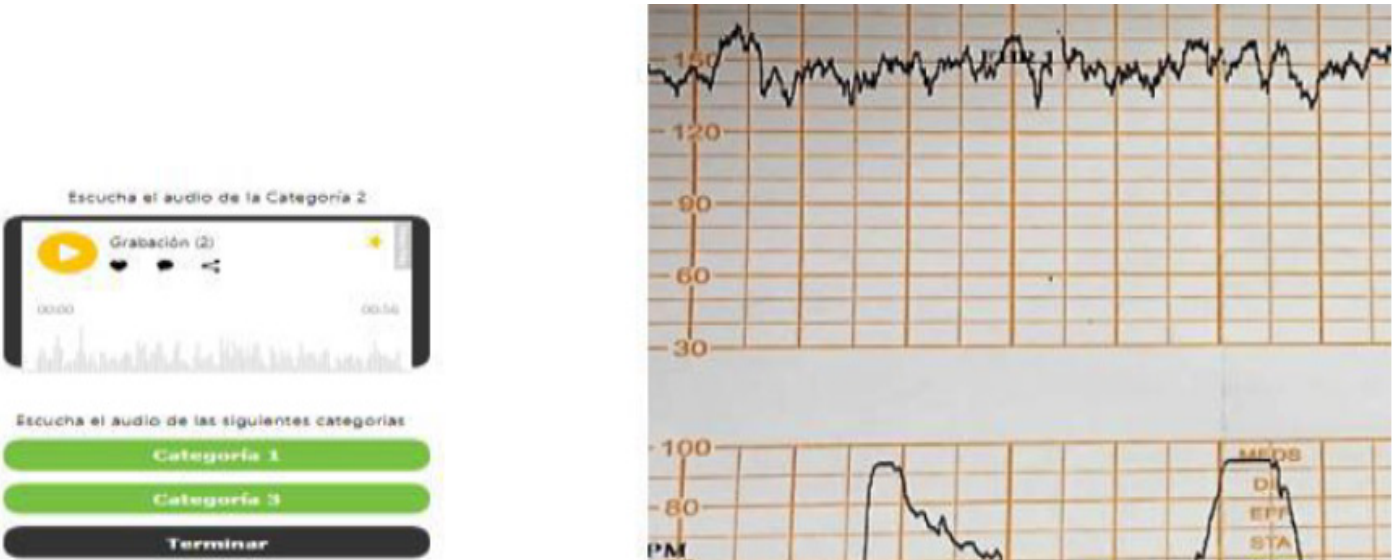

Figura 3. Sección: Trazado monitoreo electrónico materno-fetal 
Agregar Monitoreo

Selecciona el archivo del monitoreo materno fetal

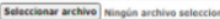

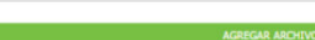

Ingrese el código del andio

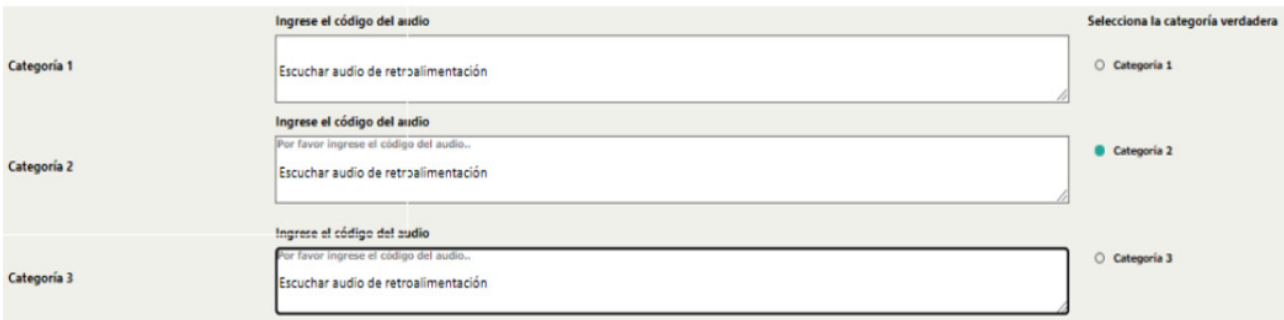

ARERNDINA

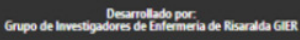

Figura 4. Sección: Categorías de interpretación monitoria materno-fetal

\section{MAEET}

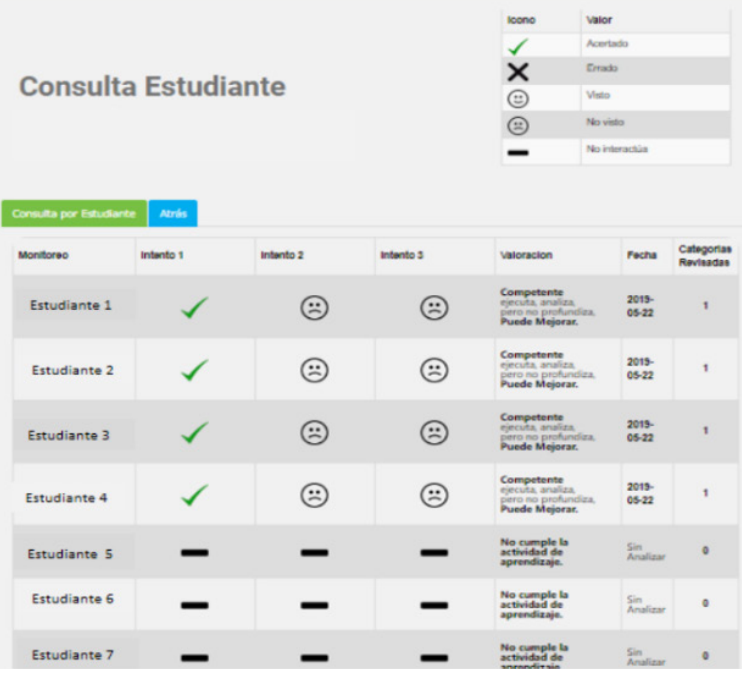

Figura 5. Sección: Retroalimentación del desempeño

\section{Evaluación de la experiencia}

En el año 2020 se realizó un pilotaje cualitativo para evaluar la facilidad del uso del prototipo y fomentar su utilización. El usuario es quien evalúa la usabilidad. Un grupo de 36 estudiantes de enfermería del área materno-perinatal, quienes utilizaron el software, respondieron voluntaria y espontáneamente entrevista abierta vía online, con respecto a los siguientes criterios de usabilidad ${ }^{32}$ : 
a) Operatividad: ¿El simulador permite que usted lo opere y controle con facilidad?

b) Inteligibilidad: ¿Le fue fácil entender la actividad o tarea que usted debe realizar?

c) Atractividad: ¿El diseño del simulador le pareció agradable y atractivo?

d) Aprendibilidad: ¿Considera que se aprende rápidamente al usar el simulador?

La información obtenida se analizó según las categorías:

- Operatividad: Los estudiantes reportaron que fue fácil acceder a los contenidos y las tareas. Algunos comentarios que ilustran la evaluación de esta categoría son: Sólo hay que seguir los pasos, se requiere tener acceso a internet, al servidor institucional, es fácil pasar de una pantalla a otra. Asimismo, los alumnos usuarios del simulador identificaron un aspecto que debe ser mejorado: El problema es que una vez que se escoge la situación de salud a realizar se debe terminar porque el sistema no deja regresar.

- Inteligibilidad: Los estudiantes expresaron: Si se tienen conocimientos previos en el tema es más fácil realizar los ejercicios, identificar y seguir cada paso que ofrece el simulador. En la medida que se interactúa con el simulador, se facilita la tarea, ya que con cada tarea ofrecida el tiempo es más corto porque ya se sabe lo que hay que hacer. Es fácil interactuar con los datos que sustentan la historia clínica o la situación de salud porque se utiliza un lenguaje claro y los registros de la monitorización electrónica fetal son de muy buena calidad.

- Atractividad: Estéticamente los estudiantes consideraron como atractivo el simulador y expresaron: Es algo real, una buena opción para lograr aprender, es agradable, llamativo, didáctico, la información es corta, pero clara. También fueron muy importantes los audios de retroalimentación que tiene el simulador porque a través de ellos es posible aclarar las dudas que a veces surgen, e igualmente el hecho de evaluar el desempeño lo pone a uno a reflexionar sobre el rol de la enfermera en la monitorización fetal. Ahora bien, los estudiantes sugirieron que [...] deberían existir más casos porque son muy pocos.

- Aprendibilidad: Los estudiantes consideraron que fue súper fácil de usar, pues sólo se necesita el link, sólo hay que seguir los pasos. Además, no tuve dificultades, súper que pueda entrar desde el celular, los ejercicios no se dejan repetir, si lo perdió, lo perdió. De igual forma, se comentó que son muy útiles los recursos multimedia y con la secuencia se aprende el uso.

La información brindada por los estudiantes mostró conceptos favorables sobre la usabilidad del simulador y permitió resaltar la necesidad de la consulta ocasional al docente, derivada del proceso reflexivo.

\section{CONCLUSIONES}

La enseñanza y el aprendizaje de la enfermería con simuladores se ha convertido no sólo en una tendencia, sino también en una necesidad apremiante e imprescindible. Como herramientas pedagógico-didácticas, la creación de escenarios de simulación, sean maniquís, robots, personas, juego de roles o simuladores computacionales, han demostrado ser benéficos para la adquisición de destrezas y habilidades propias del cuidado y desarrollo de habilidades mentales que llevan al juicio crítico y a la toma de decisiones, aspectos que cobran importancia cuando se enfrentan los escenarios reales.

El simulador computacional creado para incrementar la destreza en la interpretación del trazado toco-cardiográfico y valorar bienestar materno-fetal se consideró una herramienta de alta usabilidad. Los estudiantes identificaron facilidad de acceso, de manejo y de aprovechamiento de 
las potencialidades del equipo; asimismo, resaltaron la importancia del proceso reflexivo al que inducen, con el cual logran su aprendizaje y la necesidad de hacer la discusión del caso con el docente.

\section{RESPONSABILIDADES ÉTICAS}

Se atendieron los principios éticos registrados en la Resolución 8430 de 1993 del Ministerio de Salud y Protección Social de Colombia, en la cual se establecen las normas científicas, técnicas y administrativas para la Investigación en Salud. La metodología del proyecto no involucra riesgos bioéticos, ya que no atenta contra la integridad de los sujetos. Por el contrario, la creación del simulador computacional genera beneficios, pues busca proteger la seguridad del paciente.

La Institución Universitaria autorizó el estudio. Se obtuvo el consentimiento informado de los estudiantes que participaron en la evaluación de usabilidad, fueron informados adecuadamente de la finalidad de la evaluación a realizar y se garantizó la confidencialidad de la información.

Protección de personas y animales. Los autores declaran no haber realizado experimentos en seres humanos ni en animales para el desarrollo de esta investigación.

Conflicto de intereses. Los autores declaran no tener ningún conflicto de interés.

Financiamiento. El estudio fue autorizado y financiado por la Fundación Universitaria del Área Andina de Colombia bajo el código CVI2O2O-GIER-P12.

\section{REFERENCIAS}

1. Molinero-Bárcenas MC, Chávez-Morales U. Herramientas tecnológicas en el proceso de enseñanza- aprendizaje en estudiantes de educación superior. RIDE. Rev. Iberoam. Investig. Desarro. Educ. 2019; 10(19): 1-31. https://doi.org/10.23913/ride.v10i19.494

2. Hernández RM. Impacto de las TIC en la educación: Retos y Perspectivas. Propós. represent. 2017; 5(1): 325-47. http://dx.doi.org/10.20511/pyr2017.v5n1.149

3. Villca S. Simulación clínica y seguridad de los pacientes en la educación médica. Rev. Cien. Tec. In. 2018; 16(18): 75-88. https://bit.ly/2PzMCGM

4. Raurell-Torredà M, Gómez-Ibañez R, Miembros grupo GRISCA. La simulación de alta fidelidad: ¿Quién tiene el laboratorio más impactante?. Enferm Intensiva. 2017; 28(2): 45-7.

https://doi.org/10.1016/j.enfi.2017.04.001

5. Nunes-de Oliveira S, Lenise-Prado $M$, Silveira-Kempfer S. Utilização da simulação no ensino da enfermagem: Revisão integrativa. REME Rev Min Enferm. 2014; 18(2): 487-95.

https://doi.org/10.5935/1415-2762.20140036

6. Amaro-López L, Hernández-González PL, Hernández-Blas A, Hernández Arzola LI. La simulación clínica en la adquisición de conocimientos en estudiantes de la Licenciatura de Enfermería. Enferm. univ. 2019; 16(4): 402-13. https://doi.org/10.22201/eneo.23958421e.2019.4.543

7. Caballero-Martínez F. La simulación: El entorno virtual. Educ Méd. 2017; 18(Supl 1): 12-9. https://bit.ly/3xByTQG

8. Casal-Angulo MC. La simulación como metodología para el aprendizaje de habilidades no técnicas en Enfermería. [Tesis doctoral]. Valencia: Universitat de Valencia; 2016. https://bit.ly/3gJ8vP4

9. Altamirano-Droguett JE. La simulación clínica: Un aporte para la enseñanza y aprendizaje en el área de obstetricia. Rev. Electr. Educare. 2019; 23(2): 1-21. http://dx.doi.org/10.15359/ree.23-2.9

10. Tapia-Silva HG. Actitud hacia las TIC y hacia su integración didáctica en la formación inicial docente. Rev. actual. investig. educ. 2018; 18(3): 1-29. https://doi.org/10.15517/aie.v18i3.34437 
11. Kumar DD. Neuroscience basis of context in multimedia enhanced problem-based STEM learning. The Researcher. 2016; 1(2): 2-8. https://bit.ly/zezdzft

12. Urra-Medina E, Sandoval-Barrientos S, Irribarren-Navarro F. El desafío y futuro de la simulación como estrategia de enseñanza en enfermería. Inv Ed Med. 2017; 6(22): 119-25.

http://dx.doi.org/10.1016/j.riem.2017.01.147

13. Lanuza-Gámez FI, Rizo-Rodríguez M, Saavedra-Torres LE. Uso y aplicación de las TIC en el proceso de enseñanza- aprendizaje. Rev. cient. FAREM Estelí. 2018; (25):16-30.

http://dx.doi.org/10.5377/farem.voi25.5667

14. Posada-Morales MN, Muñoz-Astudillo MN. Prototipo Birth-Sim de atención del parto virtual: Experiencia pedagógica de aprendizaje significativo. Bogotá: Fundación Universitaria del Área Andina; 2020. https://bit.ly/2WsXyJp

15. Piana PE. Software de simulación como motivador del aprendizaje. Signos univ. 2015; 2(Extra 2): 421-32. https://bit.ly/3gPiKBy

16. Rodriguez-Zoya LG, Roggero P. La modelización y simulación computacional como metodología de investigación social. Polis. 2016; 13(39): 417-40. https://bit.ly/3aKEgDB

17. Araújo-Girão AL, Lopes-Sampaio R, Freitas-Aires S, Costa Lima-de Oliveira I, Karanini Paz-de Oliveira S, Fontenele Lima-de Carvalho RE. Medsafe: Protótipo de um jogo virtual sobre preparo e administração de medicamentos. Reme Rev Min Enferm. 2019; 23:1-8. https://doi.org/10.5935/1415-2762.20190087

18. Pirro AL. Ambientes virtuales de enseñanza y aprendizaje. El uso de simuladores. e-tramas. 2019; (3): 65-80. https://bit.ly/3nzXBwc

19. Avari M, Feizollahzadeh H, Valizadeh S, Virani F. Development and evaluation of multimedia software of vital drug prescription and calculation principles. Res Dev Med Educ. 2018; 7(1): 32-7. https://doi.org/10.15171/rdme.2018.007

20. Treviño-García VH. La estimulación multisensorial y aprendizaje. Rev. educ. incl. 2016; 9(2): $276-99$. https://bit.ly/3xA5p5X

21. Carrera-de Anda LT, Ramírez-Díaz MH. Diseño, implementación e impacto de prototipos experimentales para mejorar la enseñanza de la ley de Biot-Savart en estudiantes de ingeniería. Lat. Am. J Phys. Educ. 2017; 11(2): 1-8. https://bit.ly/zaRoqXH

22. Moreno-Pinado WE, Velázquez-Tejeda ME. Estrategia didáctica para desarrollar el pensamiento crítico. Rev. iberoam. calid. efic. cambio educ. 2017; 15(2): 53-73.

https://doi.org/10.15366/reice2017.15.2.003

23. Hernandez RM. Impacto de las TIC en la educación: Retos y Perspectivas. Propós represent. 2017; 5(1): 325-47. http://dx.doi.org/10.20511/pyr2017.v5n1.149

24. Gómez-Pawelek J. El aprendizaje experiencial. Buenos Aires: Universidad de Buenos Aires; s/f. https://bit.ly/333Pqix

25. Carrillo-Algarra AJ, Martínez-Pinto PC, Taborda-Sánchez SC. Aplicación de la filosofía de Patricia Benner para la formación en enfermería. Rev. cuba. enferm. 2018; 34(2): 1-15.

https://bit.ly/zu4VuTD

26. Terré-Rull C, Francés L. Control del bienestar fetal: Monitorización biofísica intraparto. Rev. Rol enferm. 2015; 38(2): 51-8. https://bit.ly/3vuIdUJ

27. Lutomski JE, Meaney S, Greene RE, Ryan AC, Devane D. Sistemas especializados para la evaluación fetal durante el trabajo de parto. Cochrane Database Syst Rev. 2015; (4):1-28. https://bit.ly/3f4VptT 
28. García-Gaitero F, Carrascal-Domínguez S, Renobell-Santaren V. El dibujo de la figura humana "Avatar" como elemento para el desarrollo de la creatividad y aprendizaje a través de la gamificación en Educación Primaria. Arte diseñ. ing. 2016; 5: 47-57. https://bit.ly/3gTjJjY

29. Fernández-Batalla M, Jiménez-Rodríguez ML. Documentación de casos clínicos a través de una herramienta computacional: Un estudio de credibilidad y eficiencia. Ene. 2015; 9(1).

https://dx.doi.org/10.4321/S1988-348X2015000100004

30. Mateo-Jurado I, Ricart-Marti A, Saqués-Fernández AM. Resucitación fetal intraútero. Enferm Integral. 2016; (112): 9-13

31. Canabal C, Margalef L. La retroalimentación : La clave para una evalaución orientada al aprendizaje. Profr. rev. curríc. form. profr. 2017; 21(2): 149-70. https://bit.ly/ze4mVr4

32. Perurena-Cancio L, Moráguez-Bergues M. Usabilidad de los sitios Web, los métodos y las técnicas para la evaluación. Rev. cuba. inf. cienc. salud. 2013; 24(2): 176-94. https://bit.ly/3t8dPOm 